\title{
Research on Sunglint Point Positioning Accuracy Based on Greenhouse Gas Detector
}

\author{
Jianjie Yin ${ }^{\text {a }}$, Pengmei Xu, Lizhou Hou, Long Chen and Qian Cao \\ Beijing Institute of Space Mechanics and Electricity, Visible Light Camera Research Department, China
}

\begin{abstract}
Based on greenhouse gas detector of a satellite, the operation principle, system composition, and design scheme are introduced. It emphasizes on the method of the sunglint point positioning on the detector and particularly analyses two major factors: pointing accuracy of pointing mirror and calculation accuracy of sunglint position, which have an effect on sunglint point positioning accuracy. The theoretical analysis result of sunglint point positioning accuracy is less than $6.0 \mathrm{~km}$. That is in the allowed tolerant range. Physical verification system for sunglint point positioning accuracy is built by high precision two dimensional moving platform, point source and a visible light camera which has common-path with the main optical path in the detector. It shows that the physical verification result and the theoretical analysis result to be consistent. It provides a new verification idea for positioning accuracy analysis of similarity principle aim detection.
\end{abstract}

\section{Introduction}

The information of sunglint point on the ocean is helpful and important to analyses atmospheric composition over the ocean. Usually, the sun's reflection of surface is week. When the incident angle is equal to the reflection angle, mirror reflection is occurred on the surface and the reflected light becomes strong. The reflected point is called sunglint point [1].As to detect sunglint point, the input energy enhances. Calculation of atmospheric composition and retrieval of aerosol parameters will be more accurate[2-3]. So, accurate positioning of sunglint point is an important presupposition for enhance the accuracy of atmospheric remote detection.

In order to find the sunglint point accurately and quickly and detect and analyse atmosphere over the ocean, based on an onboard greenhouse gas detector, the paper presents a calculation method of the sunglint point position in a local orbit coordinate system and verifies the sunglint positioning accuracy by theoretical analysis and physical verification.

\section{The introduction of greenhouse gas detector}

\subsection{Working principle}

\footnotetext{
a Jianjie Yin : yinjjbjut@sina.com
} 
Greenhouse gas detector is a time modulated Fouier transform interference spectrometer. It provides a atmospheric absorption spectrum of sunlight scattering by ground objects including the information of geographic and optical path to the user on orbit operation. The data array of atmospheric absorption spectrum can cover all over the world in required revisit time.

Figure. 1 shows the working principle of Greenhouse gas detector. The aiming signal is introduced by tow-dimensional pointing mirror and then goes into the interferometer module to be modulated. The modulated signal is gathered to the detector by after-optical system and colour separation and converging optical system. The detector changes the modulated optical signal to interferential electrical signal. Interferential electrical signal is amplified, filtered, quantized and encoded using the analog and digital signal processing circuit. Meanwhile, there is a visible light camera in greenhouse gas detector. The visible light camera has a common optical path with the interferential signal. But the visible light field is greater than the interferential signal field.

Sunglint point observation is an observation mode of greenhouse gas detector for atmosphere above the ocean. The detector built a mathematical model of sunglint point according to the six orbital elements and local time. It finally realized the location of sunglint point precisely through coordinate transformation and geometric conversion.

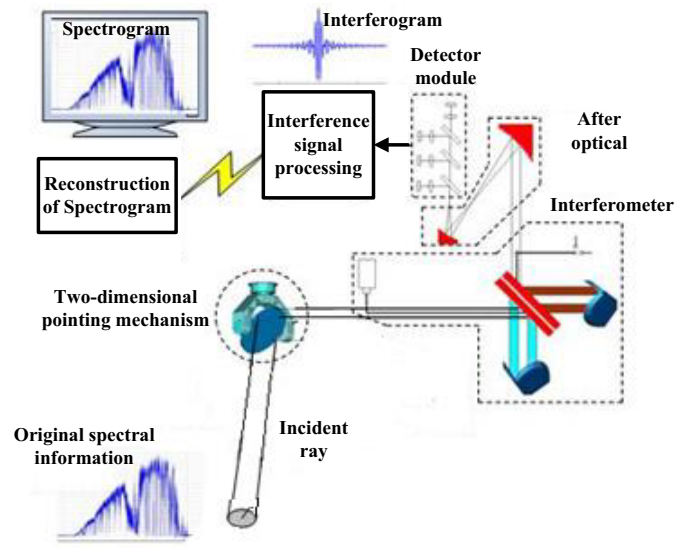

Figure 1. Schematic diagram of greenhouse gas detector.

\subsection{The method of sunglint point location}

The mathematical model of sunglint point location involves two kinds of coordinate: inertial system and orbit system [4-5]. Inertial system is that the earth center is the coordinate origin, the positive of $X$ axis is from earth center point to vernal equinox, the $\mathrm{Z}$ axis coincides with the earth's rotation axis and north is positive, the $\mathrm{Y}$ axis is perpendicular to the $\mathrm{XZ}$ plane and constitutes the right-handed coordinate system. Orbit system is that the satellite centroid is the coordinate origin, the positive of $\mathrm{X}$ axis is the flight direction of satellite orbit, the $\mathrm{Z}$ axis coincides with the line between satellite centroid and the earth center and point to the earth center is positive, the $\mathrm{Y}$ axis is perpendicular to the $\mathrm{XZ}$ plane and constitutes the right-handed coordinate system.

Figure. 2 shows the geometric relation of sun, satellite and sunglint point in inertial system. Point $\mathrm{M}$ is in the middle of the satellite vector $\boldsymbol{R}_{\text {sat }}$ and the sun vector $\boldsymbol{R}_{\text {sun. }}$ The included angle between the two vectors is $\alpha$. If $\alpha$ is divided into a value by sunglint vector $\boldsymbol{R}_{\mathrm{M}}$, that time meet the conditions of the formation of sunglint point, and the point $M$ is the sunglint point. In order to find the sunglint point $\mathrm{M}$, we can assume the existence of point $\mathrm{M}$ and finally find the point $\mathrm{M}$ equal to the incident angle $A_{\text {sunM }}$ and the reflection angle $A_{\text {satm }}$ through repeated iteration and optimization of the relationship between $A_{\text {sunM }}$ and $A_{\mathrm{satM}}$. Then we can obtain the sunglint vector $\boldsymbol{R}_{\mathrm{M}}$ in inertial system. After that, we can get the sunglint vector $\boldsymbol{S}_{\mathrm{M}}$ in inertial system through coordinate transformation [6-7]. 


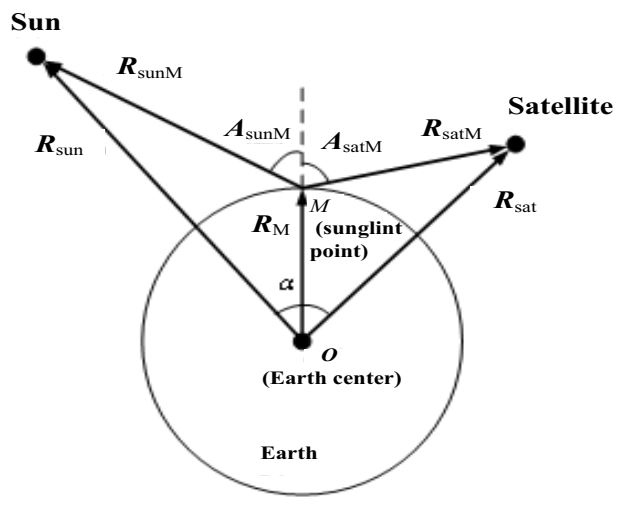

Figure.2. Geometric relation of sun, satelliate and sunglint in inertial system.

\section{Analysis of the sunglint point positioning accuracy}

\subsection{Theoretical analysis}

According to the working principle of greenhouse gas detector, the sunglint point positioning accuracy mainly consists of two parts: pointing accuracy of two-dimensional pointing mechanism and accuracy of sunglint positioning algorithm.

\subsubsection{Analysis of pointing accuracy}

The pointing accuracy of two-dimensional pointing mechanism is determined by the AT axis (along orbit direction) and CT axis (vertical orbit direction) motor and property of rotary transformer.

The AT axis is composed of a voice coil motor, pivot and rotary transformer. The step accuracy of voice coil motor is can be ignored. After quantification, the angle accuracy of the rotary transformer is $1.2^{\prime \prime}$ and the electrical error of the rotary transformer is $\pm 7.5^{\prime}$ '. The final calculation precision is $\pm 7.5^{\prime},\left( \pm 0.002^{\circ}\right)$. So the pointing accuracy of AT axis is $\pm 0.002^{\circ}$.

The CT axis is composed of a stepping motor, bearing and rotary transformer. The minimum output angle of the stepping motor is $0.036^{\circ}$. The angle accuracy of the rotary transformer is $1.2^{\prime}$ ' and the electrical error of the rotary transformer is $\pm 40^{\prime}$. The final calculation precision is $\pm 40^{\prime \prime}\left( \pm 0.01^{\circ}\right)$. So the pointing accuracy of AT axis is $\pm 0.028^{\circ}$.

\subsubsection{Analysis of sunglint point positioning algorithm accuracy}

Based on the STK model of satellite which carries with greenhouse gas detector, the sunglint point vector at 11 different time moments of one period in $\mathrm{x}, \mathrm{y}$ and $\mathrm{z}$ direction of orbit coordinate system are extracted [8-9]. It's shows in Table.1. The orbit six elements of 11 groups at the same time are as input for the sunglint point positioning algorithm. The Matlab compile results of sunglint point positioning algorithm is showed in Table.1.

Table.1. Part of results of the sunglint vector in STK and sunglint calculation

\begin{tabular}{|c|c|c|c|c|c|c|}
\hline \multirow{2}{*}{ time } & \multicolumn{3}{|c|}{$\begin{array}{c}\text { The calculation results of sunglint vector } \\
\text { exported from STK/km }\end{array}$} & \multicolumn{2}{|c|}{$\begin{array}{c}\text { The calculation results of sunglint vector } \\
\text { used by sunglint point positioning } \\
\text { algorithm in Matlab /km }\end{array}$} \\
\cline { 2 - 7 } & $x$ & $y$ & $z$ & $x$ & $y$ & $z$ \\
\hline $2016-11-821: 26: 12$ & 212.97 & -423.00 & 861.15 & 214.63 & -434.73 & 895.26 \\
\hline
\end{tabular}




\begin{tabular}{|l|c|c|c|c|c|c|}
\hline 2016-11-8 21:27:12 & 171.65 & -417.35 & 858.24 & 172.76 & -429.65 & 893.85 \\
\hline 2016-11-8 21:28:12 & 131.05 & -412.87 & 855.83 & 131.48 & -425.59 & 892.67 \\
\hline 2016-11-8 21:29:12 & 91.00 & -409.53 & 853.78 & 90.71 & -422.72 & 891.74 \\
\hline 2016-11-8 21:30:12 & 51.34 & -407.29 & 852.12 & 50.22 & -420.74 & 890.99 \\
\hline 2016-11-8 21:31:12 & 11.92 & -406.15 & 850.85 & 9.96 & -419.93 & 890.49 \\
\hline 2016-11-8 21:32:12 & -27.41 & -406.10 & 849.99 & -30.26 & -420.04 & 890.16 \\
\hline 2016-11-8 21:33:12 & -66.83 & -407.16 & 849.55 & -70.59 & -421.23 & 890.05 \\
\hline 2016-11-8 21:34:12 & -106.49 & -409.32 & 849.50 & -111.12 & -423.34 & 890.13 \\
\hline $2016-11-821: 35: 12$ & -146.55 & -412.62 & 849.89 & -152.00 & -426.50 & 890.43 \\
\hline $2016-11-821: 36: 12$ & -187.19 & -417.10 & 850.74 & -193.38 & -430.74 & 890.96 \\
\hline
\end{tabular}

For comparison conveniently, the sunglint point vectors which are exported from STK and matlab at the same time are transferred to the pointing angle $\theta$ of greenhouse gas detector. The angle $\theta$ is the include angle between sunglint vector and $\mathrm{z}$ axis. Table. 2 shows the caculation algorithm results error of sunglint point positioning is less than $0.37^{\circ}$.

Table.2. The contrast result between STK and sunglint calculation

\begin{tabular}{|c|c|c|c|}
\hline Time & $\begin{array}{c}\text { The angle calculated by } \\
\text { sunglint vectors in STK / } \\
\left({ }^{\circ}\right)\end{array}$ & $\begin{array}{c}\text { The angle calculated by } \\
\text { sunglint vectors in sunglint } \\
\text { point positioning algorithm/ } \\
\left({ }^{\circ}\right)\end{array}$ & error/ $\left(^{\circ}\right)$ \\
\hline $2016-11-821: 26: 12$ & 28.81 & 28.44 & 0.37 \\
\hline $2016-11-821: 27: 12$ & 27.73 & 27.39 & 0.35 \\
\hline $2016-11-821: 28: 12$ & 26.85 & 26.52 & 0.33 \\
\hline $2016-11-821: 29: 12$ & 26.17 & 25.87 & 0.30 \\
\hline $2016-11-821: 30: 12$ & 25.72 & 25.43 & 0.29 \\
\hline $2016-11-821: 31: 12$ & 25.53 & 25.25 & 0.27 \\
\hline $2016-11-821: 32: 12$ & 25.59 & 25.32 & 0.27 \\
\hline $2016-11-821: 33: 12$ & 25.91 & 25.63 & 0.27 \\
\hline $2016-11-821: 34: 12$ & 26.47 & 26.18 & 0.28 \\
\hline $2016-11-821: 35: 12$ & 27.26 & 26.95 & 0.31 \\
\hline $2016-11-821: 36: 12$ & 28.25 & 27.92 & 0.33 \\
\hline
\end{tabular}

\subsubsection{The results of theoretical analysis}

The ground distance of each degree of pointing mirror is $14.50 \mathrm{~km}$. So the ground field error of AT axis is $0.06 \mathrm{~km}$. The ground field error of CT axis is $0.81 \mathrm{~km}$. The ground field error caused by algorithm accuracy is $5.37 \mathrm{~km}$. Stated thus, the maximum ground field error of sunglint point detection of theoretical analysis is $6.18 \mathrm{~km}$. The sunglint point width is in $40 \sim 50 \mathrm{~km}$. this error has no effect for obtaining the sunglint point. In conclusion, the greenhouse gas detector could locate and track sunglint point effectively.

\subsection{Physical verification}




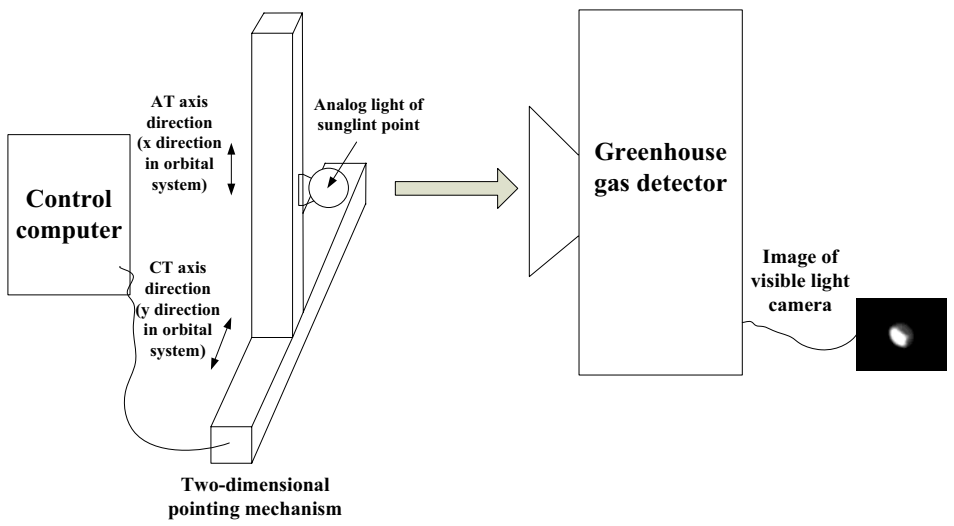

Figure.3. Schematic diagram of physical verification system.

Figure. 3 shows the schematic diagram of physical verification system. Sunglint point position data which are the same period time with the orbit six elements are translated into the displacement data in two directions of two-dimensional moving platform [10]. After to ensure the first sunglint point position in the image center of visible light camera of greenhouse gas detector, two-dimensional moving platform and greenhouse gas detector are started at the same time. It is to collect the image information of visible light camera at this period. Through analysis of the change of centroid position of light source in the image of visible light camera, sunglint point positioning accuracy of greenhouse gas detector is obtained.

The detector in visible light camera consists of $640 \times 512$ pixels. The ground pixel resolution is $0.125 \mathrm{~km}$.Figure. 4 shows all of the centroid position of light source in the image of visible light camera. The maximum distance from each centroid position of light source to the centroid position of light source of the first image is 49.20 pixels. The ground distance is $6.15 \mathrm{~km}$.

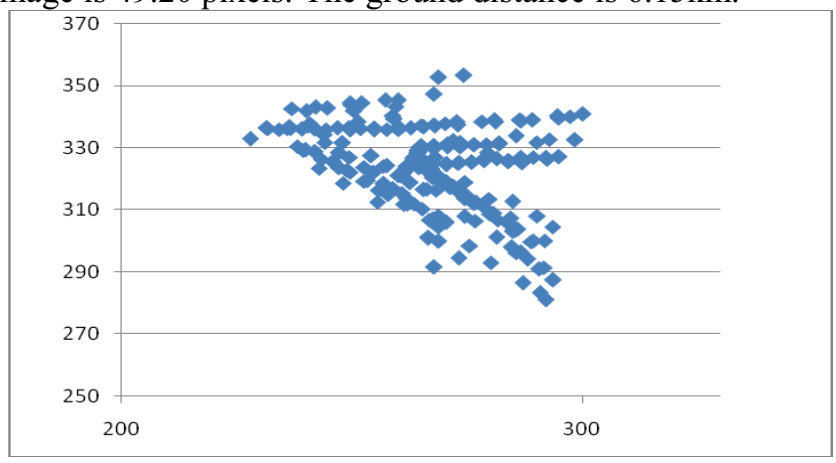

Figure.4. Distribution of light source centroid position in visible light image.

\section{Conclusion}

In conclusion, the maximum ground field error of sunglint point detection of physical verification is $6.15 \mathrm{~km}$. This result is basically consistent with the results $(6.18 \mathrm{~km})$ of theoretical analysis. Both of those analysis methods provided in this paper verified the sunglint point positioning accuracy and also verified the analysis results each other.

\section{References}

1. G.N.PLASS, G.W.KATTAWAR, J.A.GUINN. Isophotes of Sunlight Glitter in a Wind-ruffled Sea. Applied Optics, 3,643-653 (1997) 
2. SUTO.Hiroshi, KAWASHIMA.Takahiro, YOSHIDA.Jun, et al. The Pre-launch Performance Test and Calibration Results of Thermal and Near Infrared Sensor for Carbon Observation ( TANSO ) on GOSAT. Proceedings of SPIE - The International Society for Optical Engineering, 7106,71060L-1-71060L-10(2008)

3. G.INOUE, T.YOKOTA, H.OGUMA, et al. Overview of Greenhouse Gases Observing Satellite(GOSAT). American Geophysical Union Fall Meeting. San Francisco,(2004)

4. Qizhen.HU, Fang.YANG, An introduction to astronautics. Beijing,China Science and Technology Press, (2010)

5. Aoning.Xi, Wei.WANG, Near Earth Spacecraft Orbit Foundation. ChangSha,National University of Denfence Technology Press, (2003)

6. Ming.LUO, Fajie.DUAN, Xuejun.WANG, Development on Non - Contacting 3D Measurement System of Theodolites and It's Application in Aerospace. Shanghai Measurement and testing. (2009)

7. Yang.LIU, Dongyun.YI,Zhengming. WANG, Coordinate Transformation Methods from the Inertial System to the Centroid orbit System. Aerospace Control, 2,4-8(2007)

8. Suquan.DING, Bo.ZHANG, Shiyong.LIU, STK in Application of Spaceflight Simulation Analysis. BeiJing, National Defence Industry Press(2011)

9. Ying.YANG, Qi.WANG, STK in Application of Computer Simulation. BeiJing,National Defence Industry Press, (2005)

10. H.R GORDON, A.Y.MOREL, Remote Assessment of Ocean Color for Interpretation of Satellite Visible Imatery: A Review. New York,Springer-Verlag(1983) 